medications, 9(7.9\%)-H1-antagonists, 8(7\%)-alpha-agonists, 6 (5.3\%) patients-vitamin tablets, 42 (36.8\%)-other drugs*.

By observing the 928 patients aged 1-7 years old we found out the following results:cardiac medications-243 (26.2\%) patients, nonopioids-130 (14\%), antipsychotic-127 (13.7\%), antibiotics-67 (7.2\%), 361 (38.9\%)-other drugs. There were 5 suicide attempts.

The analysis of 93 patients aged 7-14 years old led to following results: nonopioids-26(28\%) patients, antipsychotic drugs-18(19.4\%), cardiac medications-14(15\%), H1-antagonists11 (11.8\%), antiemetic drugs-9 (9.7\%), 15 (16.1\%)-other drugs. There were 22 suicide attempts.

The number of 14-18 years old patients with ADIs was 125, among them $53(42.4 \%)$ patients were poisoned with nonopioids, 23(18.4\%)-antipsychotic drugs, 19(15.2\%)-H1antagonists, 12 (9.6\%)-cardiac medications, 18 (14.4\%)-other drugs. There were 91 suicide attempts.

*hormone medications, antibiotics, antifungal drugs, PPIs, PG analogues, etc.

We found out that there are 4 most common reasons for ADIs in children: non-opioid analgesics, cardiac medications, antipsychotic drugs and H1-antagonists. The first two drug classes cause predominantly ADIs in 0-7 years old children. This phenomenon might be associated with extreme curiosity of kids as well as with parental negligence. In contrast to this, children aged 7-18 overuse mostly antipsychotic drugs and $\mathrm{H} 1$-antagonists and are inclined to suicide attempts.

\section{WHAT DRUGS CAUSE INTOXICATIONS IN CHILDREN?}

Sargis Khachatryan*, E Harutyunyan, L Shahumyan, A Asatryan, H Shahumyan, A Aghajanyan, I Atoyan, A Sahakyan, G Manukyan, K Esoyan, S Mirabyan, A Sahakyan, M Grigoryan, B Baveyan, V Asoyan, H Apresyan. Yerevan State Medical University

\subsection{6/archdischild-2021-europaediatrics. 198}

In this study we aimed to investigate the most common drugs that caused ADIs in 0-18 year old patients and to observe the prevalence of suicide attempts. A retrospective study was performed using medical charts up to 7 years (2012-2018) from 'Muratsan' University hospital complex(UHC) Intensive care unit (ICU) and toxicology. We have separated the patients in four age groups $(0-1 ; 1-7 ; 7-14 ; 14-18$ years old). The overall number of patients who had ADIs and were admitted to resuscitation unit was 1260 . Mean age of patients was 4.7 years. We have included the most common drug intoxications typical for each age group.

114 patients under 1 year old have been diagnosed with ADI: 34 (29.8\%)-non-opioid analgesics, 15 (13.2\%)-cardiac medications, 9 (7.9\%)-H1-antagonists, 8 (7\%)-alpha-agonists, 6 (5.3\%) patients-vitamin tablets, 42 (36.8\%)-other drugs*. By observing the 928 patients aged 1-7 years old we found out the following results: cardiac medications-243 (26.2\%) patients, nonopioids-130 (14\%), antipsychotic-127 (13.7\%), antibiotics-67 (7.2\%), 361 (38.9\%)-other drugs. There were 5 suicide attempts.

The analysis of 93 patients aged 7-14 years old led to following results: nonopioids-26 (28\%) patients, antipsychotic drugs-18 (19.4\%), cardiac medications-14 (15\%), H1-antagonists-11 (11.8\%), antiemetic drugs-9 (9.7\%), 15 (16.1\%)-other drugs. There were 22 suicide attempts.

The number of 14-18 years old patients with ADIs was 125, among them $53(42.4 \%)$ patients were poisoned with nonopioids, 23 (18.4\%)-antipsychotic drugs, 19 (15.2\%)-H1antagonists, 12 (9.6\%)-cardiac medications, 18 (14.4\%)-other drugs. There were 91 suicide attempts. "hormone medications, antibiotics, antifungal drugs, PPIs, PG analogues, etc.

We found out that there are 4 most common reasons for ADIs in children: non-opioid analgesics, cardiac medications, antipsychotic drugs and H1-antagonists. The first two drug classes cause predominantly ADIs in 0-7 years old children. This phenomenon might be associated with extreme curiosity of kids as well as with parental negligence. In contrast to this, children aged 7-18 overuse mostly antipsychotic drugs and H1-antagonists and are inclined to suicide attempts.

\section{Paediatric Endocrinology and Diabetes}

\section{ABNORMAL TSH LEVELS AND PEDIATRIC OBESITY}

Michael Yafi* . University of Texas Health Science Center at Houston

\subsection{6/archdischild-2021-europaediatrics. 199}

To determine the prevalence of elevated thyroid-stimulating hormone (TSH) levels in obese children and adolescents referred to pediatric endocrinology clinics and its association with positive Anti Thyroid Peroxidase Antibodies. ( Anti TPO) A retrospective review of medical records of 100 obese children referred for abnormal thyroid function test was performed. Children were younger than 18 years of age with BMI above 95th percentile.

Data about age, sex, body mass index, TSH, thyroid functions, thyroid antibodies, were collected.

All patients were referred for abnormal thyroid function tests and got repeated tests along with Anti TPO levels. Interpretation of TSH results showed normal level for age in $65 \%$ and slightly elevate TSH but below $10 \mathrm{uiu} / \mathrm{ml}$ in $32 \%$ Only three obese patients (3\%) had Hashimoto disease (positive Anti TPO) and elevated TSH requiring therapy.

Obese children are often screened and referred to pediatric endocrinology for abnormal thyroid function test. It is well known that TSH levels are mildly increased in obese children but there are no evidence -based data that treating this elevation can change the outcome of obesity.

Mild elevation of TSH values in the absence of autoimmune thyroid disease is common in obese children and adolescents. This elevation is often a result of obesity rather than a cause. High Leptin level in obese children has been postulated as an etiology.

Many primary care providers and even parents are looking for 'an easy fix' of obesity by making the diagnosis of hypothyroidism and treating it.

Screening for thyroid dysfunction in obese children should be done based on symptoms and family history rather than dealing with obesity alone.

\section{TELEMEDICINE AS A METHOD OF PROVIDING PHYSICIAN COVERAGE A PILOT EXPERIENCE IN A PEDIATRIC ENDOCRINOLOGY CLINIC}

Michael Yafi*, Belinda Perzan, Michelle Rivera-Davila, Katherine Velez, Robert Yetman. University of Texas Health Science Center at Houston

10.1136/archdischild-2021-europaediatrics.200 